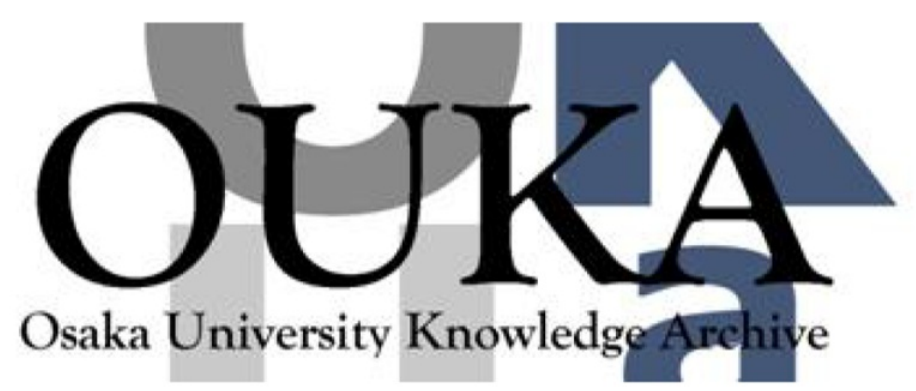

\begin{tabular}{|c|l|}
\hline Title & $\begin{array}{l}\text { Femtosecond laser manipulation of subcellular } \\
\text { organelles in Living cells }\end{array}$ \\
\hline Author(s) & $\begin{array}{l}\text { Watanabe, Wataru; Shimada, Tomoko; Itoh, } \\
\text { Kazuyoshi et al. }\end{array}$ \\
\hline Citation & $\begin{array}{l}\text { Proceedings of SPIE - The International Society } \\
\text { for Optical Eng ineering. 5863 p.58630B }\end{array}$ \\
\hline Issue Date & $2005-08-26$ \\
\hline oaire:version & VoR \\
\hline URL & $\begin{array}{l}\text { https://hdl. handle. net/11094/79145 } \\
\text { hopyright 2005 Society of Photo Optical } \\
\text { Instrumentation Engineers (SPIE). One print or } \\
\text { electronic copy may be made for personal use } \\
\text { only. Systematic reproduction and distribution, } \\
\text { duplication of any material in this publication } \\
\text { for a fee or for commercial purposes, or } \\
\text { modification of the contents of the publication } \\
\text { are prohibited. }\end{array}$ \\
\hline rights \\
\hline Note & \begin{tabular}{l} 
\\
\hline
\end{tabular} \\
\hline
\end{tabular}

Osaka University Knowledge Archive : OUKA

https://ir. Library. osaka-u. ac. jp/

Osaka University 


\title{
Femtosecond laser manipulation of subcellular organelles in living cells
}

\author{
Wataru Watanabe, Tomoko Shimada, and Kazuyoshi Itoh \\ Department of Material and Life Science, Graduate School of Engineering, Osaka University, \\ 2-1, Yamadaoka, Suita, Osaka 565-0871, Japan \\ watanabe@photonics.mls.eng.osaka-u.ac.jp \\ http://www-photonics.mls.eng.osaka-u.ac.jp/ \\ Sachihiro Matsunaga and Kiichi Fukui \\ Department of Biotechnology, Graduate School of Engineering, Osaka University \\ 2-1, Yamadaoka, Suita, Osaka 565-0871, Japan
}

\begin{abstract}
Manipulation - that is, inactivation and movement - of subcellular organelles in living cells is important to understand cell dynamics. We review disruption of subcellular organelles in living cells by tightly focusing femtosecond laser pulses inside the cells. Photodisruption of organelles in living cells was experimentally confirmed by restaining of organelles. Femtosecond laser based nanosurgery has the possibility to remove or manipulate organelles in living cells and may lead to elucidation of functional interactions between organelles, protein transportation, and inter-cell communication.
\end{abstract}

Keywords: femtosecond laser, HeLa cell, organelles, mitochondria, disruption, inactivation, nanosurgery, fluorescence microscopy

\section{INTRODUCTION}

Studying intact organelles and their function in a living cell is essential to understand cell dynamics. It is difficult to directly reveal the characteristics of organelles in a targeted cell in vivo by conventional molecular and biochemical techniques; optical methods, however, can manipulate or modify the behavior of intact organelles in living cells. For example, optical tweezers using infrared lasers have allowed us to manipulate cells, organelles, and subcellular particles. [1] With optical tweezers, however, the trapping force strongly depends on the shape and refractive index of the trapped particle. A focused continuous-wave or long-pulse ultraviolet (UV) and visible laser beam has been used for microsurgery of organelles (Fig. 1). Dissection and inactivation of subcellular organelles in plant and animal cells were demonstrated with submicron spatial resolution by laser-induced plasma formation [2-6]. However, continuous-wave or long-pulse lasers in UV and visible regions have some disadvantages, namely, low light penetration depth, collateral damage outside the focal volume, and the possibility of photodamage to living cells due to absorption. In addition, UV radiation is also known to be responsible for oxidative stress leading to apoptosis.

Femtosecond lasers operating in the near infrared (NIR) region have attractive advantages compared with conventional UV lasers, such as efficient two-photon ionization, no out-of-focus absorption, and the absence of significant transfer of heat or mechanical energy to surrounding structures due to nonlinear optical effects around the focal volume. A two-photon microscope using NIR femtosecond laser pulses was developed to observe subcellular structures [7]. The high penetration depth of the NIR region provides the possibility of optical sectioning in deep tissue. Due to the lack of out-of-focus photodamage and photobleaching, two-photon NIR microscopy appears to be a safe tool having no adverse effects on cellular metabolism, reproduction, and viability [8]. However, above a certain threshold energy, it is reported that photodamage occurs in the cells[9-15].

Therapeutic Laser Applications and Laser-Tissue Interactions II, edited by Hubert van den Bergh, Alfred Vogel, Proc. of SPIE-OSA Biomedical Optics, SPIE Vol. 5863 @ 2005 SPIE and OSA · 1605-7422/05/\$15 


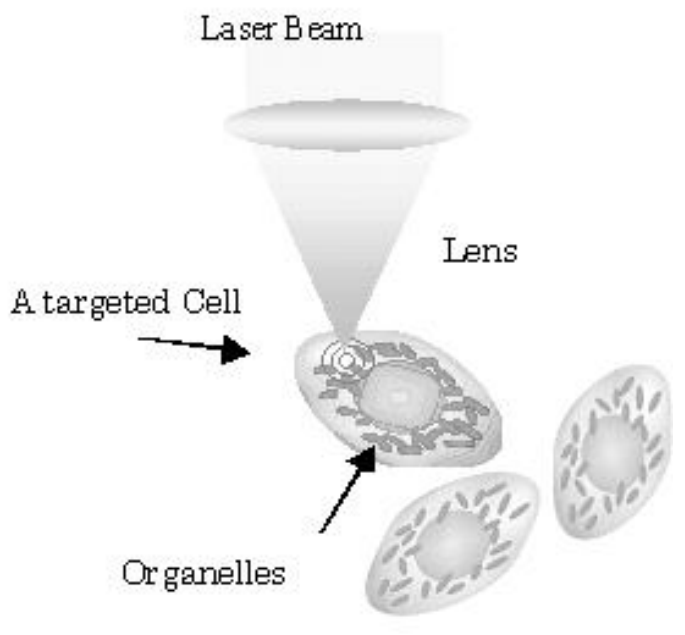

Fig. 1: The focused laser beam can structurally modify or inactivate the function of targeted organelles within a targeted living cell with high spatial resolution on the nanometer to micrometer scale.

When NIR femtosecond laser pulses are tightly focused, the intensity in the focal volume can become high enough to result in a permanently damaged region in the cell with sub-micron size (Fig. 2). When intense femtosecond laser pulses are tightly focused at the targeted organelles inside a cell, the intensity in the focal volume can become high enough to cause nonlinear absorption, through multiphoton, tunneling and avalanche ionization processes. The free electrons absorb energy from the electromagnetic field of the laser pulse, leading to laser-induced optical breakdown, and the generation of high-density plasma. The increase in electron density leads to absorption of the femtosecond laser pulse and the high-density plasma expands with hypersonic velocity that drives a shockwave. High-density plasma and the shockwave play important roles in photodisruption of organelles. Several groups have reported NIR laser-based nanoprocessing. König et al. performed dissection of chromosomes [16] and DNA transfection [17] with NIR femtosecond lasers. A single organelle was completely knocked out without disturbing surface layers and affecting the adjacent organelles or the viability of both plant cells and animal cells [18,13]. Supatto et al. have shown that an alloptical approach can be used both to locally disrupt the structural integrity of tissues inside live embryos by using NIR femtosecond laser ablation combined with multimodal nonlinear microscopy [19]. They demonstrated controlled intravital ablations preserving local cytoskeleton dynamics and resulting in the modulation of specific morphogenetic movements in nonmutant Drosophila embryos. A quantitative description of complex movements is obtained both in GFP-expressing systems by using whole-embryo two-photon microscopy and in unlabeled nontransgenic embryos by using third harmonic generation microscopy. Yanik et al. demonstrated femtosecond laser axotomy [20]. They cut the circumferential axons (labeled with green fluorescent protein) inside C. elegans by using pulse energies of 10-40 nanojoules at a repetition rate of $1 \mathrm{kHz}$ and tightly focused, 200-fs, NIR laser pulses. Dyefilling of axotomized neurons confirmed that the observed axon gaps are not due to photobleaching, but to physical disconnection of the axons and they observed functional recovery associated with nerve regeneration. Sacconi $e t$ al. used NIR femtosecond laser pulses for a combination of microscopy and nanosurgery on fluorescently labeled structures within living cells [21]. Threedimensional reconstructions of microtubule structures tagged with green fluorescent protein (GFP) are made during different phases of the cell cycle. Further, the microtubules are dissected using the same laser beam but with a higher laser power than for microscopy. They showed that the nanosurgery on the structures labeled with GFP is most likely due to two-photon absorption of GFP molecules. Shen et al. demonstrated knocking out of individual mitochondria in a living cell without disturbing the rest of the cell on a scale of a few hundred nanometers using tightly focused NIR femtosecond laser pulses at a low repetition rate $(1 \mathrm{kHz})$ [22]. Heisterkamp et al. carried out a study of the energy dependence of the ablation of fluorescently-labeled subcellular structures in the cytoskeleton and nuclei of fixed endothelial cells using femtosecond laser pulses focused through a highnumericalaperture objective lens (1.4 NA) [23]. They find that the energy threshold for photobleaching lies between 0.9 and $1.7 \mathrm{~nJ}$. By comparing the changes in 
fluorescence with the actual material loss determined by electron microscopy, we find that the threshold for true material ablation is about $20 \%$ higher than the photobleaching threshold to about 3 nJ. NIR laser-based nanoprocessing of cellular structures without compromising the viability of cells has potential applications in cell biology because one can control functional interactions between organelles and inter-cell communication only at targeted locations. In this paper, we review the manipulation of subcellular organelles in living cells by tightly focusing NIR femtosecond laser pulses. We present the experimental confirmation of photodisruption of subcellular organelles in a living cell by tightly focusing NIR femtosecond laser pulses inside the cell [24].

\section{EXPERIMENTAL SETUP}

Figure 3 shows a schematic diagram of the experimental setup [24]. To visualize organelles in a living cell, a confocal laser scanning microscope was used with He-Ne laser (wavelength $543 \mathrm{~nm}$ ) or an Ar-ion laser (wavelength $488 \mathrm{~nm}$ ). The laser beam is focused in the cell through the objective lens; a water-immersion objective (NA 1.2) whose chromatic aberration was optimized for the visible and NIR region for short-term observation, or an oil-immersion objective (NA 1.4) for long-term observation. Scanning of the focused laser beams in the xy plane was accomplished by a pair of highspeed galvanometer mirrors. The back-propagated one-photon fluorescence was collected using the same objective and epi-detected with a photomultiplier tube to record the fluorescence intensities point by point to form two-dimensional confocal cross-sectional images at a certain depth inside the cell. The depth scanning was achieved by moving the objective with a stepping motor to obtain three-dimensional confocal images.

Irradiation with femtosecond laser pulses was performed using a regeneratively amplified Ti:sapphire laser system which produced $150-\mathrm{fs}, 800-\mathrm{nm}, 1-\mathrm{kHz}$ pulses. The beam was directed into the scanning system through DM1 and DM2. The laser pulses were focused at the desired location of an organelle inside the cell. The pulse energy was reduced by inserting neutral density filters. First, one-photon fluorescence images of cells were obtained by excitation with cw He-Ne laser or $\mathrm{Ar}+$ laser. Next, we set the focal point of the objective lens to a target organelle inside the cells. By opening the shutter, femtosecond laser pulses were focused into the target. Finally, after femtosecond laser irradiation, one-photon fluorescence images of the cells were obtained by excitation with the cw He-Ne laser or $\mathrm{Ar}+$ laser.

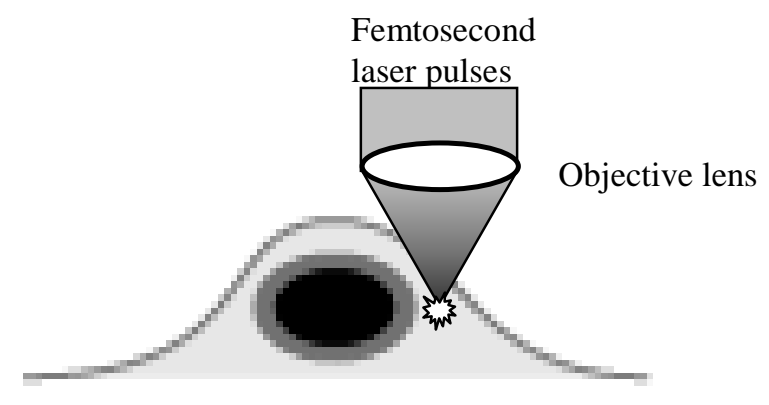

Fig. 2: Schematic of femtosecond laser nanosurgery.

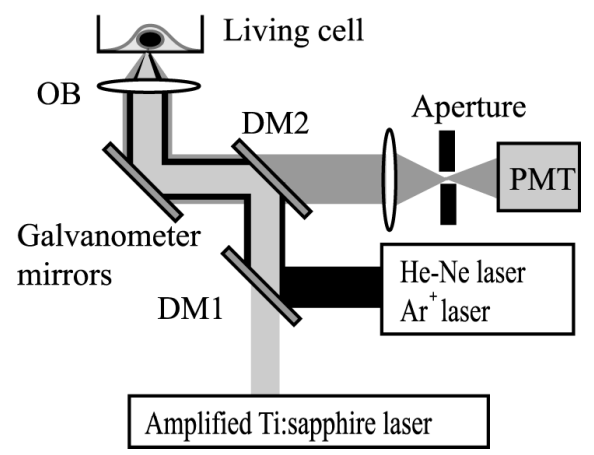

Fig. 3: Schemtic diagram of the experimental setup. L, lens; OB, objective lens; DM1 and DM2, dichroic mirrors; PMT, photomultiplier tube. 


\section{DISRUPTION OF MITOCHONDRIA}

We show the disruption of individual mitochondria in living HeLa cells. Mitochondria in the HeLa cells were stained with MitoTracker Red. Figures 4(a) and (b) show the confocal images of HeLa cells obtained before and after femtosecond laser irradiation. Figures 4(c) and (d) show magnified views of the square areas indicated in Figs. 4(a) and (b), respectively. Femtosecond laser pulses at an energy of $3 \mathrm{~nJ} /$ pulse were focused with the water-immersion objective at a mitochondrion indicated by the arrow in Fig. 4(a), or at the center of the circle in Fig. 4(c). Figure 4 shows that fluorescence from a single mitochondrion within a size of $1 \mu \mathrm{m}$ disappeared. Neighboring mitochondria were not photodisrupted or photobleached even though they were only a few micrometers away. The positional difference of the mitochondria outside the focal region between the before and after images was attributed to cytoplasmic streaming, which indicated the viability of the cells.

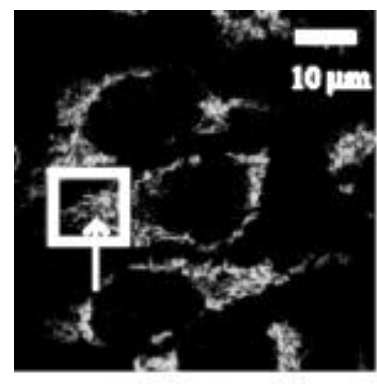

(a)

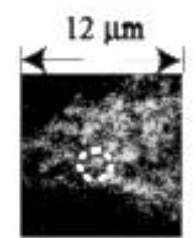

(c)

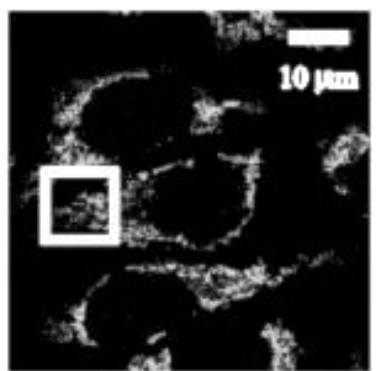

(b)

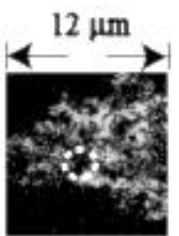

(d)

Fig. 4: Confocal images of HeLa cell before and after femtosecond-laser irradiation. (a) and (b) confocal images of the HeLa cell before and after femtosecond laser irradiation. Red fluorescence shows mitochondria of HeLa cells stained with MitoTracker Red. A target mitochondrion is indicated by a white arrow. (c) and (d) magnified views of square areas indicated in (a) and (b), respectively. The center of the dotted circles show target mitochondria.

In the next experiment, the cells were transfected with an EYFP fused with a mitochodria-targeted sequence of cytochrome $\mathrm{C}$ oxidase. Before the irradiation of femtosecond laser pulses, one-photon fluorescence images of cells were observed by excitation with the $\mathrm{Ar}^{+}$laser at a wavelength of $488 \mathrm{~nm}$, as shown in Fig. 5(a). Femtosecond laser pulses were focused into a target mitochondrion at an energy of $3 \mathrm{~nJ} /$ pulse. Figure 5(b) shows that fluorescence from a single mitochondrion within a size of a few $\mu \mathrm{m}$ disappeared. Neighboring mitochondria were not photodisrupted or photobleached. The disruption of mitochondria is independent of the fluorescence labeling. 


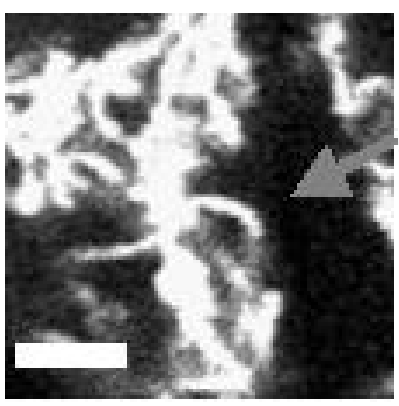

$3 \mu \mathrm{m}$

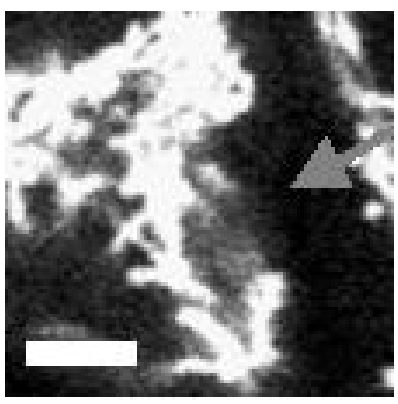

$3 \mu \mathrm{m}$

(a)

(b)

Fig. 5: (a) Confocal images of laser-irradiated cells before and after restaining. Yellow fluorescence shows mitochondria visualized by EYFP before femtosecond laser irradiation. (b) confocal image obtained after femtosecond laser irradiation.

\section{RESULTS AFTER RESTAINING}

We performed an experiment on restaining of the cells, to investigate whether the absence of fluorescence intensity was due to disruption or due to bleaching of the fluorescence. In this experiment, the cells were transfected with an EYFP fused with a mitochodria-targeted sequence of cytochrome $\mathrm{C}$ oxidase. One-photon fluorescence images of cells were observed by excitation with the $\mathrm{Ar}^{+}$laser at a wavelength of $488 \mathrm{~nm}$, as shown in Fig. 6(a). Figure 6(c) shows a magnified view of the square area indicated in Fig. 6(a). Femtosecond laser pulses at an energy of $3 \mathrm{~nJ} / \mathrm{pulse}$ were focused with the oil-immersion objective (NA, 1.4). Figure 6(b) shows the confocal image obtained after fs laser irradiation and Fig. 6(d) shows a magnified view of the square area indicated in Fig. 6(b). No fluorescence from the surrounding mitochondria in a region a few microns wide from an irradiated mitochondrion was observed. We restained the mitochondria with MitoTracker Red after femtosecond laser irradiation. If the mitochondria were not disrupted, the remaining mitochondria would be detected with MitoTracker Red within the irradiation area. MitoTracker Red was added to the medium immediately after femtosecond laser irradiation. Because mitochondria were normally stained 30 minutes after addition of MitoTracker Red, confocal images obtained 35 minutes after addition of MitoTracker Red are shown in Fig. 6(e) and (f). Figure 6(e) shows the confocal image obtained by excitation with the Ar ${ }^{+}$laser, whereas Fig. 6(f) shows the confocal image obtained by excitation with the He-Ne laser. The figures show that the mitochondria in the laser-irradiated region were not restained after addition of MitoTracker Red. 


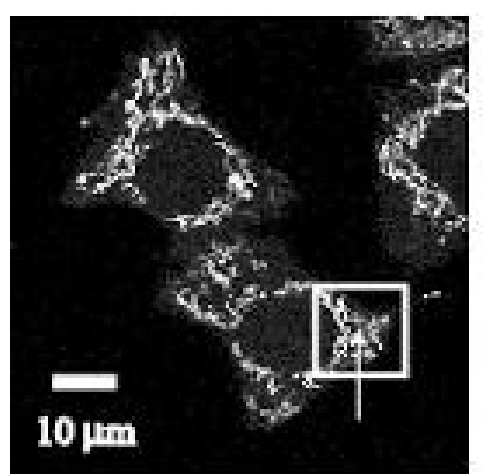

(a)

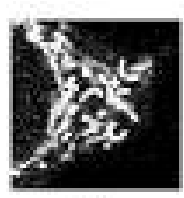

(c)

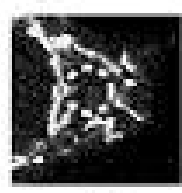

(d)

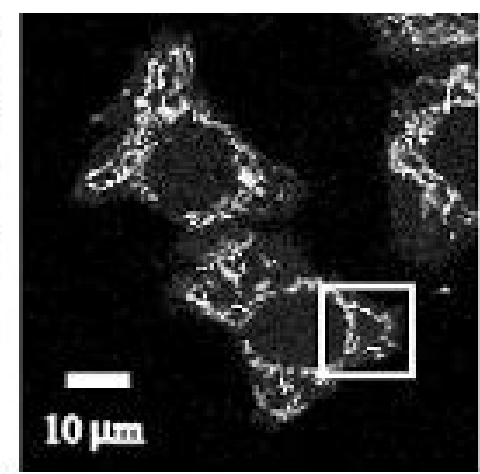

(b)

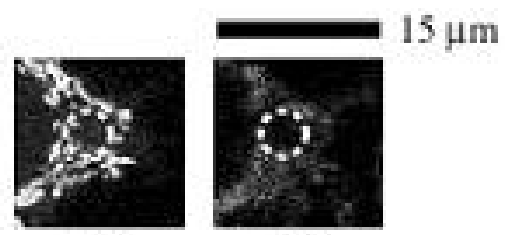

(f)

Fig. 6:(a) Confocal images of laser-irradiated cells before and after restaining. Yellow fluorescence shows mitochondria visualized by EYFP. Target mitochondrion is indicated by a white arrow. (c) magnified view of square area indicated in (a) before femtosecond laser irradiation. (b) confocal image obtained after femtosecond laser irradiation. (d) magnified view of square area in (b). (e) and (f) confocal images obtained after restaining by MitoTracker Red. (e) confocal image obtained by excitation with the Ar+ laser. (f) confocal image obtained by excitation with the He-Ne laser. Dotted circles show target mitochondria.

\section{CONCLUSION}

We present the feasibility of NIR femtosecond laser pulses to manipulate subcellular organelles in living cells. Photodisruption of organelles in living cells with focused femtosecond laser pulses was experimentally confirmed by restaining organelles after irradiation by focused femtosecond laser pulses, and by stacking three-dimensional confocal images without affecting the viability of the cells. This femtosecond laser disruption technique has possible applications in the removal of specific organelles in living cells and may provide information on function and dynamics of organelles in living cells and lead to the elucidation of functional interactions between organelles and inter-cell communication.

\section{REFERENCES}

1. A. Ashkin, K. Shutze, J. M. Dziedzic, U. Euteneuer, and M. Schliwa, "Force generation of organelle transport measured in vivo by an infrared laser trap," Nature 348, 346-348 (1990).

2. R. L. Amy and R. Storb, "Selective mitochondrial damage by a ruby laser microbeam: an electron microscope study," Science 150, 756-757 (1965).

3. M. W. Berns, J. Aist, J. Edwards, K. Strahs, J. Girton, P. McNeil, J. B. Rattner, M. Kitzes, M. Hammerwilson, L. H. Liaw, A. Siemens, M. Koonce, S. Peterson, S. Brenner, J. Burt, R. Walter, P. J. Bryant, D. Vandyk, J. ouclombe, T. Cahill, and G. S. Bern, "Laser microsurgery in cell and developmental biology," Science 213, 505-513 (1981).

4. M. W. Berns, W. H. Write, and R. W. Steubing, "Laser microbeam as a tool in cell biology," Int. Rev. Cytol. 129, 1-44 (1991). 
5. K. O. Greulich, Micromanipulation by light in biology and medicine, (Birkhähser-Verlag, Basel, Switzerland, 1999).

6. V. Venygopalan, A. Guerra III, K. Hahen, and A. Vogel, "Role of laser-induced plasma formation in pulse cellular microsurgery and micromaniplation," Phys. Rev. Lett. 88, 078103 (2002).

7. W. Denk, J. H. Strickler, and W. W. Webb, "Two-photon laser scanning fluorescence microscopy," Science 248, 73-76 (1990).

8. J. M. Squirell, D. L. Wokosin, J. G. White, and B. D. Bavister, "Long-term two-photon fluorescence imaging of mammalian embryos without compromising vitality," Nat. Biotechnolo. 17, 763-767 (1999).

9. Y. Sako, A. Sekihata, Y. Yanagisawa, M. Yamamoto, Y. Shimada, K. Ozaki, and A. Kusumi, "Comparison of twophoton excitation laser scanning microscopy with UV-confocal laser scanning microscopy in three-dimensional calcium imaging using the fluorescence indicator Indo-1, J. Microsc. 185, 9-20 (1997).

10. K. König, P. T. C. So, W. W. Mantulin, B. J. Tromberg, and E. Gratton, "Cellular response to near-infrared femtosecond laser pulses in two-photon microscopes," Opt. Lett. 22, 135-136 (1997).

11. K. König, T. W. Becker, P. Fischer, I. Riemann, and K. -J. Halbhuber, "Pulse-length dependence of cellular response to intense near-infrared laser pulses in multiphoton microscopes," Opt. Lett. 24, 113-115 (1999).

12. H. J. Kester, D. Baur, R. Uhl, and S. W. Hell, " $\mathrm{Ca}^{2+}$ fluorescence imaging with pico- and femtosecond two-photon excitation: signal and photodamage," Biophys. J. 77, 2226-2236 (1999).

13. K. König, "Laser tweezers and multiphoton microscopes in life sciences," Histochem. Cell Biol. 114, 79-92 (2000).

14. H. Oehring, I. Riedmann, P. Fisher, K. -J. Halbhuber, and K. König, "Ultrastructure and reproduction behaviour of single CHO-K1 cells exposed to near infrared femtosecond laser pulses," Scanning 22, 263-270 (2000).

15. U. K. Tirlapur, K. König, C. Peuckert, R. Krieg, and K. Halbhuber, "Femtosecond near-infrared laser pulse elicit generation of reactive oxygen species in mammalian cells leading to apotosis-like death," Exp. Cell Res. 263, 88-97 (2001).

16. K. König, W. Riemann, and W. Fritzsche, "Nanodissection of human chromosomes with near-infrared femtosecond laser pulses," Opt. Lett. 26, 819-821(2001).

17. U. K. Tirlapur and K. König, "Targeted transfection by femtosecond laser," Nature 418, 290-291 (2002).

18. U. K. Tirlapur and K. König "Femtosecond near-infrared laser pulses as a versatile non-invasive tool for intratissue nanoprocessing in plants without compromising viability," The Plant J. 31, 365-374 (2002).

19. M. F. Yanik, H. Cinar, H. N. Cinar, A. D. Chisholom, Y. Jin, and A. Ben-Yakar, "Functional regeneration after laser axotomy", Nature 432, 8222 (2004).

20. W. Supatto, D. Débbarre, B. Moulia, E. Brouzébs J.-L. Martin, E. Farge, and E. Beaurepaire, "In vivo modulation of morphogenetic movements in Drosophila embryos with femtosecond laser pulses," Proc. Natl. Acad. Sci. USA. 102, 1047-1052 (2005).

21. L. Sacconi, I. M Tolić-Nørrelykke, R. Antolini, and F. S. Pavone, "Combined intracellular three-dimensional imaging and selective nanosurgery by a nonlinear microscope," J. of Biomed. Opt. 10, 014002 (2005).

22. N. Shen, D. Datta, C. B. Schaffer, P. LeDuc, D. E. Ingber, E. Mazur, "Ablation of cytoskeletal filaments and mitochondria in cells using a femtosecond laser nanoscissor," Mechanics and Chemistry of Biosystems 2, 17-26 (2005).

23. A. Heisterkamp, I. Z. Maxwell, E. Mazur, J. M. Underwood, J. A. Nickerson, S. Kumar, and D. E. Ingber, "Pulse energy dependence of subcellular dissection by femtosecond laser pulse," Opt. Express (accepted).

24. W. Watanabe, N. Arakawa, S. Matsunaga, T. Higashi, K. Fukui, K. Isobe, and K. Itoh, "Femtosecond laser photodisruption of sub-cellular organelles in a living cell," Opt. Express 12, 4203-4213 (2004). 